\title{
Efficacy of Combined XingZhi-YiNao Granules and Hyperbaric Oxygen Therapy for Cognition and Motor Dysfunction in Patients with Delayed Encephalopathy after Acute Carbon Monoxide Poisoning
}

\author{
Li Qin, ${ }^{1}$ Chu Meihua, ${ }^{1}$ Guo Dadong, ${ }^{2}$ Wang Li, ${ }^{1}$ \\ Wang Jinglin, ${ }^{3}$ Ding Xiaoyu, ${ }^{1,4}$ Bi Mingjun, ${ }^{3}$ and Zou Yong ${ }^{1}$ \\ ${ }^{1}$ Department of Integration of Chinese and Western Medicine, The Affiliated Yantai Yuhuangding Hospital of Qingdao University, \\ Yantai, Shandong 264000, China \\ ${ }^{2}$ Eye Institute of Shandong University of Traditional Chinese Medicine, Jinan, Shandong 250002, China \\ ${ }^{3}$ Emergency Center, The Affiliated Yantai Yuhuangding Hospital of Qingdao University, Yantai, Shandong 264000, China \\ ${ }^{4}$ Department of Clinical Medicine, Qingdao University Medical College, Qingdao, Shandong 266003, China
}

Correspondence should be addressed to Li Qin; liqin701015@163.com, Bi Mingjun; bimingjun00@163.com, and Zou Yong; ytmpzy@sina.com

Received 8 July 2017; Accepted 26 October 2017; Published 26 November 2017

Academic Editor: Caigan Du

Copyright (c) 2017 Li Qin et al. This is an open access article distributed under the Creative Commons Attribution License, which permits unrestricted use, distribution, and reproduction in any medium, provided the original work is properly cited.

Purpose. To investigate the efficacy of XingZhi-YiNao (XZYN) granules and hyperbaric oxygenation (HBO) for cognition and motor dysfunction in patients with delayed encephalopathy after acute carbon monoxide poisoning (DEACMP). Methods. Eightynine patients with DEACMP were randomly divided into control group $(n=19)$, HBO group $(n=32)$, and XZYN group $(n=38)$. All patients received conventional treatment. HBO group received HBO therapy once daily. XZYN group received extra XZYN granules plus $\mathrm{HBO}$ treatment. The related indexes including activity of daily living (ADL) scale, Montreal cognitive assessment (MoCA) scale, and mini mental state examination (MMSE) scale were measured. Cerebral white matter injury, age related white matter changes (ARWMC) scale, and the amplitude and latency of P300 were assessed. Results. Compared with control group, the neurological function scores of $\mathrm{ADL}, \mathrm{MoCA}$, and MMSE in HBO and XZYN groups were significantly improved, the impairment degree of brain white matter and cognition function were obviously alleviated, the latencies of P300 were significantly shortened, and the amplitudes of P300 were evidently increased $(P<0.05)$. Treatment efficacy of XZYN group was superior to that of HBO group $(P<0.05)$. Conclusion. Combined XZYN granules and HBO can significantly improve cognition and motor functions in patients with DEACMP.

\section{Introduction}

Nowadays, carbon monoxide (CO) poisoning is the most common health problem for people in many countries [1]. It can lead to a lot of neurophysiology and neuropathological changes, among which acute brain injury and delayed encephalopathy after carbon monoxide poisoning (DEACMP) are the most common neurological complications [2]. Multiple factors have been found to be associated with the development of DEACMP, such as ischemia and hypoxia, cytotoxic injury, reperfusion injury, immune dysfunction, and neurotransmitter dysregulation, resulting in overlapping processes $[3,4]$. Nevertheless, the specific mechanisms of DEACMP are still unclear. Currently, mounting evidence suggests hyperbaric oxygen ( $\mathrm{HBO}$ ) therapy as the best treatment for patients with acute $\mathrm{CO}$ poisoning, but its effectiveness for the prevention and treatment in DEACMP remains controversial $[5,6]$ because approximately $10 \%$ to $30 \%$ patients finally develop DEACMP despite successful HBO treatment of the initial symptoms during one session or repetitive sessions. In terms of the medicine application, more and more studies have paid attention to corticosteroids [7], 
antioxidants, and free radical scavengers $[8,9]$. Other drugs, such as brain-protective agents, traditional Chinese medicine (TCM), and natural animal and plant extracts [10-12], have also been applied to clinical practice. However, these drugs can not fundamentally prevent or inhibit the occurrence and development of either acute brain damage or DEACMP due to the lack of evidence-based medicine. It has been demonstrated that the early application of TCM can improve brain microcirculation, promote nerve cell survival, and benefit neurological recovery [13]. Therefore, TCM may play a role in repairing the structure and function of damaged nerve cells, improving the neurological function in patients with severe acute CO poisoning and DEACMP.

Based on the manifestation of patients with DEACMP, we fabricated the formulation of "XingZhi-YiNao (XZYN) granules" using a therapeutic method of "detoxifying, promoting blood circulation, dissolving phlegm, removing obstruction, unraveling wisdom, and replenishing essence." The XZYN granules include 8 types of boil-free granules of traditional Chinese medicine extracts as follows: Coptis Rhizome, $10 \mathrm{~g}$ (Batch Number: 1701081, Anhui Puren Chinese Medicine Slices Co., Ltd.); Panax Ginseng, 10 g (Batch Number: 160501, Hubei Didao Medicinal Materials Technology Co., Ltd.); Poria cum Radix Pini, $10 \mathrm{~g}$ (Batch Number: 161111, Anhui Puren Chinese Medicine Slices Co., Ltd.); Acorus tatarinowii Schott, $20 \mathrm{~g}$ (Batch Number: 1707092, Anhui Puren Chinese Medicine Slices Co., Ltd.); Milkworts, 10 g (Batch Number: 1704262, Anhui Puren Chinese Medicine Slices Co., Ltd.); Salvia miltiorrhiza, 30 g (Batch Number: 1707103, Anhui Puren Chinese Medicine Slices Co., Ltd.); Radix Puerariae, $10 \mathrm{~g}$ (Batch Number: 1703042, Anhui Puren Chinese Medicine Slices Co., Ltd.); Bile Arisaema, $10 \mathrm{~g}$ (Batch Number: 160503, Sichuan Fuzheng Pharmaceutical Co., Ltd.). All boil-free granules were purchased from Shandong green leaf Medical \& Pharmaceutical Co., Ltd. (Shandong, China). The identification for each granule was determined by thin layer chromatography (Qingdao Haiyang Chemical Co. Ltd., Qingdao, China), and the quality of all products met the requirements of Chinese Pharmacopeia [14]. In this study, we aimed to explore the efficacy of TCM in patients with DEACMP through retrospective case series analysis, offering a foundation for the clinical application of TCM in CO poisoning.

\section{Material and Methods}

2.1. Recruited and Excluded Criteria. Patients diagnosed with DEACMP were recruited in the Affiliated Yantai Yuhuangding Hospital of Qingdao University from June 2011 to May 2016. The criteria for DEACMP were as follows: (1) history of CO exposure; (2) age from 18 to 75 years; (3) clinical symptoms of DEACMP occurring after 2 60 days of "latent period" without any clinical manifestation of acute CO poisoning; (4) demyelination lesions of bilateral cerebral white matter in brain MRI; (5) patients and their family members signing informed consent and completing all of the treatment program and a follow-up plan. In this study, those who had chronic hepatic and renal insufficiency, diabetes, coronary heart disease, cerebrovascular disease, malignant tumor, drug poisoning or addiction, radioactive encephalopathy, encephalitis, multiple sclerosis, sarcoidosis, mild cognitive impairment, or dementia diagnosed before the poisoning onset or midway death or failure to complete follow-up planning were excluded from the final statistics. Follow-up examinations were performed from 2011 to 2017.

2.2. Participants and Treatment Strategy. A total of 112 patients with DEACMP were recruited at first as the primary endpoint. Before treatment, 23 cases were excluded because they did not agree to randomization or failed to complete the follow-up. Finally, 89 patients were selected to participate in the study and then randomly divided into a control group ( $n$ $=19$ ), a hyperbaric oxygen treatment group (HBO group, $n=$ 32), and a XingZhi-YiNao granules treatment group (XZYN group, $n=38$ ). For every patient, the detailed information associated with the demographic and clinical parameters, such as sex, age, body mass index (BMI), education level (years), work type, $\mathrm{CO}$ exposure time, latent phase, coma time, blood carboxyhemoglobin level, S100B protein level, and lactate clearance rate (\%), was listed in Table 1 . No statistical differences were found among the three groups before treatment $(P>0.05)$. Meanwhile, all patients received nasal tube of oxygen inhalation and conventional treatment with regulating water and electrolytes, maintaining stable blood pressure and blood glucose, and injecting cytidine diphosphate choline (CDPC) and vitamin B. Patients in HBO group underwent extra hyperbaric oxygen therapy once daily till 2 months, while the cases in XZYN group received XingZhi-YiNao granules twice daily based on the treatment options of $\mathrm{HBO}$ group. Clinical manifestations of the patients were recorded by two trained neurologists, and laboratory studies, including blood routine, blood lipid, blood glucose, electrolytes and liver and kidney function, brain MRI, and neural electrophysiology, were performed before and within 72 hours after treatment in order to understand the unpleasant side effects induced by drugs.

\subsection{Experimental Methods}

2.3.1. Neurological Function Assessment. The movement and cognition changes of patients were measured by activities of daily living (ADL) scale, Montreal cognitive assessment (MoCA) scale, and mini mental state examination (MMSE) before and after treatment at 1 month and 2 months, respectively. The total ADL score was set to 100, and the higher the score, the better the ability of daily life of patients. In the meantime, the total MMSE and MoCA scores were both set to 30 , and the higher score indicated the stronger cognitive ability of patients. However, a low score $(<26)$ was regarded as cognitive dysfunction. The neurological function assessment of all patients was evaluated by two trained raters in a doubleblind manner at the same time point. We described our continuous data by their mean value and standard deviation or median (interquartile range) in the final statistics.

2.3.2. Neuroimaging and White Matter Lesions Score. All patients underwent routine MRI scanning of brain on a 
TABLE 1: Baseline characteristics and clinical data of the included patients.

\begin{tabular}{|c|c|c|c|c|c|}
\hline Variable & Control group & HBO group & XZYN group & $q / \chi^{2}$ & $P$ value \\
\hline Number & 19 & 32 & 38 & & \\
\hline Female/male & $10 / 9$ & $18 / 14$ & $21 / 17$ & 0.0062 & $P>0.05$ \\
\hline Age (y) & $47.2 \pm 7.5$ & $48.5 \pm 6.8$ & $47.6 \pm 8.2$ & $1.213 \sim 1.835$ & $P>0.05$ \\
\hline BMI $\left(\mathrm{kg} / \mathrm{m}^{2}\right)$ & $22.3 \pm 3.5$ & $21.7 \pm 3.2$ & $22.5 \pm 3.7$ & $0.758 \sim 1.136$ & $P>0.05$ \\
\hline Education level (y) & $10.1 \pm 4.2$ & $9.8 \pm 4.4$ & $9.2 \pm 4.9$ & $0.892 \sim 2.015$ & $P>0.05$ \\
\hline Work type $(\mathrm{Me} / \mathrm{Ma})$ & $12 / 7$ & $22 / 10$ & $25 / 13$ & 0.1869 & $P>0.05$ \\
\hline CO exposure time $(\mathrm{h})$ & $9.6 \pm 7.2$ & $10.8 \pm 6.8$ & $10.1 \pm 7.5$ & $1.270 \sim 1.753$ & $P>0.05$ \\
\hline COHb levels (\%) & $28.0 \pm 12.5$ & $25.7 \pm 11.2$ & $26.9 \pm 13.2$ & $1.305 \sim 2.281$ & $P>0.05$ \\
\hline Latent phase $(\mathrm{d})$ & $15.2 \pm 7.6$ & $13.5 \pm 7.4$ & $15.8 \pm 8.1$ & $1.208 \sim 1.913$ & $P>0.05$ \\
\hline Coma time $(\mathrm{h})$ & $6.8 \pm 3.6$ & $7.0 \pm 3.2$ & $6.5 \pm 3.9$ & $1.422 \sim 2.148$ & $P>0.05$ \\
\hline Serum S100B $(\mu / \mathrm{L})$ & $0.8 \pm 0.3$ & $0.7 \pm 0.2$ & $0.8 \pm 0.5$ & $1.270 \sim 1.695$ & $P>0.05$ \\
\hline Lactate clearance rate $(\%)$ & $39.3 \pm 6.2$ & $38.7 \pm 7.1$ & $37.9 \pm 6.4$ & $1.369 \sim 2.512$ & $P>0.05$ \\
\hline
\end{tabular}

BMI: body mass index; Me: mental worker; Ma: manual worker; $\mathrm{COHb}$ : carboxyhemoglobin.

Philips Intera system (Philips Medical Systems, Best, the Netherlands). At $1.5 \mathrm{~T}$, an axial 2D spin echo sequence with the following sequence parameters was acquired: repetition time/echo time/inversion time (TI)/flip angle $(\mathrm{FA})=$ $540 \mathrm{~ms} / 14 \mathrm{~ms} / \mathrm{ms} / 90^{\circ}$, matrix $=256 \times 256,22$ slices, thickness $=5 \mathrm{~mm}$, and spacing at $6 \mathrm{~mm}$. The white matter lesions were evaluated by the age related white matter change (ARWMC) scale [15] and the revised scale of Xiong et al. [16] as described in Table 2. The total scale score was 30 . The higher the score, the more serious the white matter lesions and the wider the damage scope. All scores were performed by two physicians simultaneously in a double-blind manner, and the average value was calculated and expressed in the final statistics.

2.3.3. P300 Detection. The latency and amplitude of P300 were recorded using a electromyography evoked potentiometer of Japanese Photoelectric MEB-9200K. All tests were performed in a double-blind manner by two experienced professionals.

2.4. Statistical Analysis. All statistical analyses were performed using SPSS17.0 statistical software, and all the continuous variables were expressed as mean \pm standard deviation. Analysis of covariance (ANCOVA) was conducted to observe the difference effect of treatments on neurological function scores among three groups. Student's test and the chi-squared test were applied to investigate the differences between the two groups on demographic and baseline clinical variables. All procedures were 2 -tailed and a value of $P<0.05$ was considered statistical for all tests.

\section{Results}

3.1. Comparisons of ADL, MoCA, MMSE, and ARWMC Scores among Three Groups. Based on the statistical results, we found that there was no significant difference in ADL, MoCA, and MMSE scores among three groups prior to treatment $(P>0.05)$. However, the scores of ADL, MoCA, and MMSE in both $\mathrm{HBO}$ group and XZYN group were significantly higher than those in control group after treatment $(P<$ 0.05). Moreover, compared with those in HBO group, the scores of ADL, MoCA, and MMSE in XZYN group were apparently elevated at the same time point (Table $3, P<0.05$ ). This result suggested that hyperbaric oxygen can improve the daily exercise ability and cognitive function of patients with DEACMP, and the efficacy of combined HBO and XZYN granules is more effective in patients with DEACMP.

Imaging data showed that many abnormal performances could be identified in patients with DEACMP to some extent, mainly as follows: long T1, long T2 signals in MRI, slightly higher signal in FLAIR, and high signal in DWI, with low signal in diffusion coefficient (ADC). Brain lesion often lies in cerebral cortex of occipital lobe, semioval center, white matter around lateral ventricle, and globus pallidus and could be observed in white matter and deep gray matter nucleus. Some coexistence or fusion of multiple lesions could also be showed in part of subjects with DEACMP. There was no significant difference in ARWMC score among three groups before treatment $(P>0.05)$. The ARWMC score was slightly decreased at 1 month after intervention treatment in both HBO and XZYN groups, but no statistical difference was found as compared with NC group $(P>0.05)$. The score of ARWMC was significantly lower in both $\mathrm{HBO}$ and $\mathrm{XZYN}$ groups at 2 months than in control group (Table 3, $P<0.05)$, suggesting that hyperbaric oxygen therapy can significantly reduce the lesion degree of white matter in patients after CO poisoning, and the combined application of TCM and hyperbaric oxygen therapy may be more conducive to neurological recovery in patients with DEACMP.

3.2. Comparison of Event-Related Potential P300 among Three Groups before and after Treatment. Event-related potentials (P300) is an objective index to reflect the severity and the prognosis judgment of patients with cognitive impairment $[17,18]$. In the present study, we noted that, before treatment, there were no significant differences in the latency and amplitude of P300 among three groups $(P>0.05)$. However, the latency of P300 in patients was significantly 
TABLE 2: Operational definitions of ARWMC scale.

\begin{tabular}{lcc}
\hline Score & Frontal lobe, parietal-occipital lobe, temporal lobe, and infratentorial region & Basal ganglia \\
\hline 0 & No lesions (PVWMC: symmetrical, well-defined ventricle, or DWMC $<5 \mathrm{~mm})$ & No lesions \\
1 & Focal lesions (PVWMC, nonsymmetrical caps or bands $<5 \mathrm{~mm}$, or DWMC between 5 and $9 \mathrm{~mm})$ & One focal lesion $(>5 \mathrm{~mm})$ \\
2 & Beginning confluence of lesions (PVWMC between 5 and $10 \mathrm{~mm}$ or DWMC between 10 and & More than one focal lesion \\
& $25 \mathrm{~mm}$ or connecting bridge between two focal lesions) & Confluence of lesions \\
3 & Diffuse involvement of most region (PVWMC $>10 \mathrm{~mm}$ or & DWMC $>25 \mathrm{~mm})$
\end{tabular}

TABLE 3: Comparison of ADL, MoCA, MMSE, and ARWMC at different time points before and after treatment in each group.

\begin{tabular}{|c|c|c|c|c|c|}
\hline Group & $n$ & $\mathrm{ADL}$ & $\mathrm{MoCA}$ & MMSE & ARWMC \\
\hline \multicolumn{6}{|l|}{ Control group } \\
\hline Before treatment & 19 & $12.4 \pm 5.8$ & $15.1 \pm 3.5$ & $14.1 \pm 4.3$ & $18.7 \pm 4.1$ \\
\hline 1 month after treatment & 19 & $21.5 \pm 6.6$ & $16.7 \pm 4.0$ & $15.9 \pm 3.0$ & $17.5 \pm 3.2$ \\
\hline 2 months after treatment & 19 & $23.0 \pm 6.1$ & $18.2 \pm 3.6$ & $18.1 \pm 4.5$ & $15.2 \pm 3.3$ \\
\hline \multicolumn{6}{|l|}{$\mathrm{HBO}$ group } \\
\hline Before treatment & 24 & $13.8 \pm 6.0$ & $14.6 \pm 3.9$ & $13.7 \pm 4.6$ & $19.0 \pm 3.3$ \\
\hline 1 month after treatment & 24 & $46.9 \pm 7.7^{\mathrm{a}}$ & $20.5 \pm 3.0^{\mathrm{a}}$ & $18.0 \pm 3.2^{\mathrm{a}}$ & $16.1 \pm 3.1$ \\
\hline 2 months after treatment & 24 & $60.5 \pm 8.1^{\mathrm{a}}$ & $22.2 \pm 2.7^{\mathrm{a}}$ & $22.4 \pm 3.5^{\mathrm{a}}$ & $8.7 \pm 2.2^{\mathrm{a}}$ \\
\hline \multicolumn{6}{|l|}{ XZYN group } \\
\hline Before treatment & 24 & $12.9 \pm 5.1$ & $15.5 \pm 4.2$ & $14.4 \pm 3.8$ & $18.1 \pm 3.9$ \\
\hline 1 month after treatment & 24 & $57.6 \pm 6.4^{\mathrm{ab}}$ & $23.3 \pm 2.9^{\mathrm{ab}}$ & $21.4 \pm 3.5^{\mathrm{ab}}$ & $13.2 \pm 3.3^{\mathrm{ab}}$ \\
\hline 2 months after treatment & 24 & $70.2 \pm 8.3^{\mathrm{ab}}$ & $26.1 \pm 3.1^{\mathrm{ab}}$ & $25.9 \pm 4.1^{\mathrm{ab}}$ & $7.0 \pm 2.1^{\mathrm{ab}}$ \\
\hline
\end{tabular}

shortened and the amplitude was significantly higher after treatment with combined hyperbaric oxygen and XZYN granules, especially at 1 month, accompanied by a statistical difference compared with the control group (Table $4, P<$ 0.05). We also found that the P300 latency in XZYN group was significantly shorter and the amplitude was higher than that of $\mathrm{HBO}$ group at the same time point $(P<$ 0.05 ), suggesting that hyperbaric oxygen therapy combined with XZYN granules can significantly improve the cognitive function of patients with delayed encephalopathy after CO poisoning.

3.3. Adverse Events. No apparent adverse reactions of the drugs were found in the included patients. There were no significant alterations for blood glucose, blood lipids, and liver and kidney function during the whole treatment session.

\section{Discussion}

DEACMP is the most common neurological complication after acute $\mathrm{CO}$ poisoning. The main manifestations are dyskinesia, advanced intelligence loss, and mental behavior abnormality, which seriously affect the life quality of the patients. However, the detailed pathogenesis of DEACMP is unclear. Most scholars believed that ischemia and hypoxia, neuronal apoptosis and necrosis, the increased excitatory amino acid, neurotransmitter abnormalities, oxidative stress, and lipid peroxidation products caused by $\mathrm{CO}$ poisoning could lead to the structural and functional changes of myelin basic proteins, the abnormal expression of myelin-derived axonal growth inhibitors (such as Nogo, NgR, and Ogmp), and the imbalanced-expression of cytokines and inflammatory molecules in the central nervous system, which may be an important factor of generalized demyelination lesions in white matter of brain tissue $[19,20]$. ADL, MoCA, and MMSE are the most common indicators to evaluate the functions of movement and cognition of patients. ARWMC is mainly used for the quantitative evaluation of white matter lesions and easy to operate with good credibility. Its characteristic is that it can rapidly and quantitatively judge the size and severity of the lesions in white matter and basal ganglia area. Our results showed that the scores of ADL, MoCA, and MMSE in patients with DEACMP were significantly lower than the average, while the extensive demyelinating lesions were found in brain tissue in all three groups before treatment, and the score of ARWMC was obviously increased, suggesting that the patients had obvious motor and cognitive dysfunctions. However, after the comprehensive treatment of combined hyperbaric oxygen and XZYN granules, the motor and cognitive functions of patients were improved significantly; some demyelinating lesions were recovered. This evidently indicated the reversibility of motor and cognitive disabilities and white matter lesions in DEACMP patients to a certain extent $[2,21]$. The treatment of combined hyperbaric oxygen 
TABLE 4: Comparison of event-related potential P300 among three groups.

\begin{tabular}{|c|c|c|c|c|c|c|c|}
\hline \multirow[b]{2}{*}{ Group } & \multirow[b]{2}{*}{$n$} & \multicolumn{3}{|c|}{ Latency of P300 } & \multicolumn{3}{|c|}{ Amplitude of P300 } \\
\hline & & $\begin{array}{c}\text { Before } \\
\text { treatment }\end{array}$ & $\begin{array}{c}1 \text { month after } \\
\text { treatment }\end{array}$ & $\begin{array}{c}2 \text { months after } \\
\text { treatment }\end{array}$ & $\begin{array}{c}\text { Before } \\
\text { treatment }\end{array}$ & $\begin{array}{c}1 \text { month after } \\
\text { treatment }\end{array}$ & $\begin{array}{c}2 \text { months after } \\
\text { treatment }\end{array}$ \\
\hline Control group & 19 & $410.3 \pm 32.5$ & $390.2 \pm 27.5$ & $381.7 \pm 30.3$ & $3.8 \pm 1.1$ & $4.0 \pm 1.0$ & $4.1 \pm 1.5$ \\
\hline HBO group & 24 & $407.6 \pm 30.9$ & $373.6 \pm 28.7^{\mathrm{a}}$ & $352.5 \pm 23.6^{\mathrm{a}}$ & $3.7 \pm 1.6$ & $4.5 \pm 0.9^{\mathrm{a}}$ & $5.6 \pm 1.3^{\mathrm{a}}$ \\
\hline XZYN group & 24 & $412.1 \pm 34.0$ & $370.4 \pm 25.3^{\mathrm{a}}$ & $332.9 \pm 20.4^{\mathrm{ab}}$ & $3.7 \pm 1.3$ & $5.3 \pm 1.2^{\mathrm{ab}}$ & $6.5 \pm 1.6^{\mathrm{ab}}$ \\
\hline
\end{tabular}

Note. Compared with the control group at the same time point, ${ }^{\mathrm{a}} \mathrm{P}<0.05$; compared with $\mathrm{HBO}$ group at the same time point, ${ }^{\mathrm{b}} \mathrm{P}<0.05$.

and XZYN granules achieved better efficacy in DEACMP patients in our study, and it is worth further clinical use.

Event-related potential P300 is an important indicator for brain cognitive and psychological state, and participants were required to engage in cognitive activities, including attention, alertness, working memory, executive function, and many other cognitive functions. Currently, the determination of P300 has been widely used in clinical practice. However, the amplitude of P300 is also affected by a variety of stimulus parameters, such as the probability, the order and the quality of stimulation, the difficulty of the task, the sensitivity of the reward, and the emotional input of the subjects. The increased amplitude of P300 may reflect an urgent adaptation and regulatory processing. In the present study, we found that the latency of $\mathrm{P} 300$ was prolonged and the amplitude was decreased in patients with DEACMP to some extent. Statistical analysis revealed that the latency of P300 was related to the scores of MoCA and MMSE in DEACMP patients, suggesting that P300 latency could be used as an important index to judge the cognitive function of patients. The latency of P300 is associated with MoCA and MMSE scores in DEACMP patients, suggesting that P300 latency can be used as an important index to judge the cognitive function of patients. However, the relationship between P300 latency and the scores of ADL and ARWMC is uncertain in our study. We suspect that it is mainly concerned with the following aspects: (1) P300 is a complex waveform, which can objectively reflect the nerve fiber contact in cerebral cortex and cognitive activity participation in different brain areas, and is closely related to memory, cognitive, calculation, understanding, judgment, and reasoning functions. P300 latency reflects the speed of neural activity and processing, relating to the intensity of attention, memory, and cognitive function. The ADL score mainly evaluates the daily life ability of the patients, and the state of consciousness, the mental state, the cognitive function, and the limb movement condition of the patients can result in the decrease of ADL score. ARWMC is currently used to assess the severity and extent of white matter lesions. Based on the aforementioned results, we found that white matter lesions in DEACMP patients were common in the cortex of parietal and occipital lobe, semioval center, white matter around lateral ventricle, and globus pallidus, and there were different degrees of damage in the white matter and deep gray nuclei. When nerve fiber demyelination and other ARWMC typical changes occur in these brain areas, these will slow or block information transmission of cortical neurons, which ultimately led to signal disruption of different brain functional areas, resulting in cognitive dysfunction. However, individuals with DEACMP exhibited comprehensive cognitive dysfunction, and the clinical manifestations slightly varied during each session of the disease. The memory, language, and the visual function of DEACMP patients were rapidly declined in a very short term, and personality abnormalities and other cognitive functions (including generalization, judgment, and multiability in analyzing and solving questions) appeared at a later stage and seriously and often accompanied with emotional disorders and extrapyramidal and pyramidal system dysfunction, which may directly affect the cognitive activities of the participants involved in the study. This can explain why P300 is related to the scores of ADL and ARWMC. (2) All patients were enrolled voluntarily and were required to complete all the trials. And thus, those who failed to cooperate with the research or lost following up or had suffered either serious illness or halfway death were excluded from the statistics. Thus, a relatively smaller sample size, younger population, and milder poisoning were included as compared with the general DEACMP patients and thus may affect the accuracy of the study.

\section{Conclusions}

In summary, this study revealed that the combined application of traditional Chinese medicine XingZhi-YiNao granules and $\mathrm{HBO}$ could significantly improve cognitive function and motor function in patients with delayed encephalopathy after acute carbon monoxide. However, it needs to verify and support the efficacy of this method from more patients and further research in the future.

\section{Abbreviations}

ADL: $\quad$ Activity of daily living

ARWMC: Age related white matter changes

CO: $\quad$ Carbon monoxide

$\mathrm{COHb}: \quad$ Carboxyhemoglobin

DEACMP: Delayed encephalopathy after acute carbon monoxide poisoning

HBO: Hyperbaric oxygenation

MMSE: Mini mental state examination

MoCA: Montreal cognitive assessment

TCM: Traditional Chinese medicine. 


\section{Ethical Approval}

The study was approved by the institutional review board of Qingdao University and registered.

\section{Consent}

All subjects (or first-degree relative) gave their written consent.

\section{Conflicts of Interest}

The authors declare that they have no conflicts of interest.

\section{Acknowledgments}

This work was supported by the National Natural Science Foundation of China (no. 81571283), the Natural Science Foundation of Shandong (nos. ZR2016HL21, ZR2017LH068), the Traditional Chinese Medicine Science and Technology Development Project in Shandong (no. 2015-420), and the Medical and Health Development Project Grants in Shandong (no. 2015WS0020). The authors are thankful to Dr. Hai Kang in the Affiliated Yantai Yuhuangding Hospital of Qingdao University and Dr. Wu-Lian in Binzhou Medical University for constant support during this study.

\section{References}

[1] "Centers for disease control and prevention (CDC). Carbon monoxide exposures-United States, 2000-2009," Morbidity and Mortality Weekly Report, vol. 60, no. 30, pp. 1014-1017, 2011.

[2] A. F. Geraldo, C. Silva, D. Neutel, L. L. Neto, and L. Albuquerque, "Delayed leukoencephalopathy after acute carbon monoxide intoxication," Journal of Radiology Case Reports, vol. 8, no. 5, pp. 1-8, 2014.

[3] S. R. Thom, V. M. Bhopale, D. Fisher, J. Zhang, and P. Gimotty, "Delayed neuropathology after carbon monoxide poisoning is immune-mediated," Proceedings of the National Acadamy of Sciences of the United States of America, vol. 101, no. 37, pp. 13660-13665, 2004.

[4] J. A. Guzman, "Carbon Monoxide Poisoning," Critical Care Clinics, vol. 28, no. 4, pp. 537-548, 2012.

[5] M. G. Senol, S. Yildiz, D. Ersanli et al., "Carbon monoxideinduced cortical visual loss: treatment with hyperbaric oxygen four years later," Medical Principles and Practice, vol. 18, no. 1, pp. 67-69, 2009.

[6] G. Garrabou, J. M. Inoriza, C. Morén et al., "Mitochondrial injury in human acute carbon monoxide poisoning: the effect of oxygen treatment," Journal of Environmental Science and Health - Part C Environmental Carcinogenesis and Ecotoxicology Reviews, vol. 29, no. 1, pp. 32-51, 2011.

[7] W. Xiang, H. Xue, B. Wang et al., "Combined application of dexamethasone and hyperbaric oxygen therapy yields better efficacy for patients with delayed encephalopathy after acute carbon monoxide poisoning," Drug Design, Development and Therapy, vol. 11, pp. 513-519, 2017.
[8] K. Mori, T. Beppu, Y. Fujisawa et al., "Effect of free radical scavenger, edaravone, for patients with carbon monoxide poisoning," Clinical Neurology and Neurosurgery, vol. 139, pp. 5661, 2015.

[9] Q. Li, M. J. Bi, W. K. Bi, H. Kang, L. J. Yan, and Y.-L. Guo, "Edaravone attenuates brain damage in rats after acute CO poisoning through inhibiting apoptosis and oxidative stress," Environmental Toxicology, vol. 31, no. 3, pp. 372-379, 2016.

[10] H. Wang, Y. Li, Q. Wu, C. Xu, and Q. Liu, "Combination of butylphthalide with umbilical mesenchymal stem cells for the treatment of delayed encephalopathy after carbon monoxide poisoning," Medicine, vol. 95, no. 49, article no 5412, 2016.

[11] M.-J. Bi, X.-N. Sun, Y. Zou et al., "N-Butylphthalide improves cognitive function in rats after carbon monoxide poisoning," Frontiers in Pharmacology, vol. 8, article no. 64, 2017.

[12] M. J. Bi, Q. Li, D. D. Guo et al., "Sulphoraphane improves neuronal mitochondrial function in brain tissue in acute carbon monoxide poisoning rats," Basic \& Clinical Pharmacology \& Toxicology, vol. 120, pp. 541-549, 2017.

[13] K. Sun, J. Fan, and J. Han, "Ameliorating effects of traditional chinese medicine preparation, chinese materia medica and active compounds on ischemia/reperfusion-induced cerebral microcirculatory disturbances and neuron damage," Acta Pharmaceutica Sinica B, vol. 5, no. 1, pp. 8-24, 2015.

[14] Chinese Pharmacopeia, "China Medical Science Press, Beijing, China, 2010".

[15] L. O. Wahlund, F. Barkhof, F. Fazekas et al., "A new rating scale for age-related white matter changes applicable to MRI and CT," Stroke, vol. 32, no. 6, pp. 1318-1322, 2001.

[16] Y. Xiong, J. Yang, A. Wong et al., “Operational definitions improve reliability of the age-related white matter changes scale," European Journal of Neurology, vol. 18, no. 5, pp. 744-749, 2011.

[17] M. Lu, N. Doñamayor, T. F. Münte, and J. Bahlmann, "Eventrelated potentials and neural oscillations dissociate levels of cognitive control," Behavioural Brain Research, vol. 320, pp. 154164, 2017.

[18] T. M. Davis, B. D. Hill, K. J. Evans et al., "P300 eventrelated potentials differentiate better performing individuals with traumatic brain injury: a preliminary study of semantic processing," The Journal of Head Trauma Rehabilitation, vol. 32, no. 4, pp. E27-E36, 2017.

[19] Y. Yang, Y. Liu, P. Wei et al., "Silencing Nogo-A promotes functional recovery in demyelinating disease," Annals of Neurology, vol. 67, no. 4, pp. 498-507, 2010.

[20] Q. Li, Y. Cheng, M. J. Bi et al., "Effects of N-Butylphthalide on the expressions of $\mathrm{Nogo} / \mathrm{NgR}$ in rat brain tissue after carbon monoxide poisoning," Environmental Toxicology and Pharmacology, vol. 39, no. 2, pp. 953-961, 2015.

[21] T. Tapeantong and N. Poungvarin, "Delayed encephalopathy and cognitive sequelae after acute carbon monoxide poisoning: Report of a case and review of the literature," Journal of the Medical Association of Thailand, vol. 92, no. 10, pp. 1374-1379, 2009. 


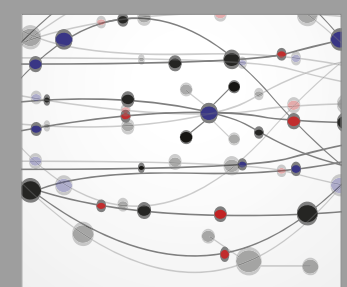

The Scientific World Journal
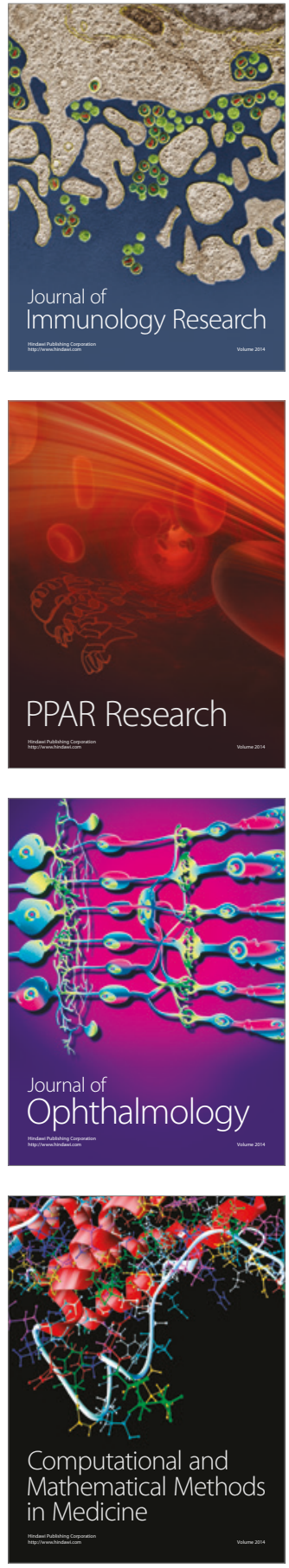

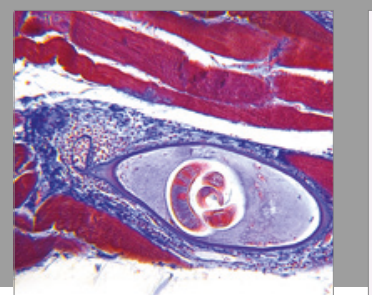

Gastroenterology Research and Practice
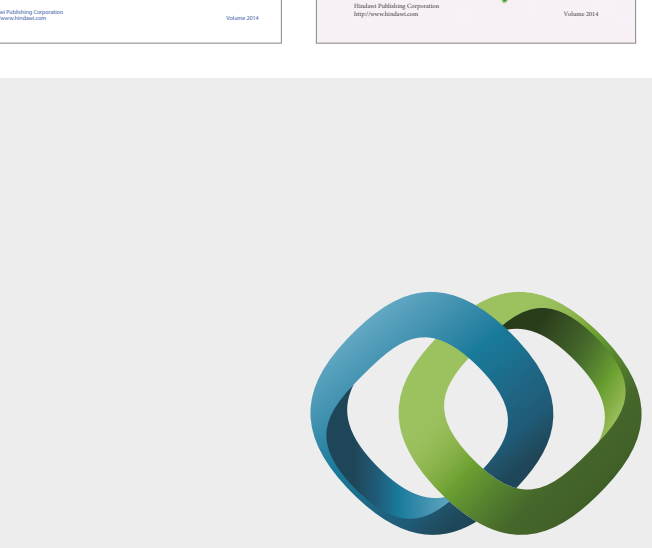

\section{Hindawi}

Submit your manuscripts at

https://www.hindawi.com
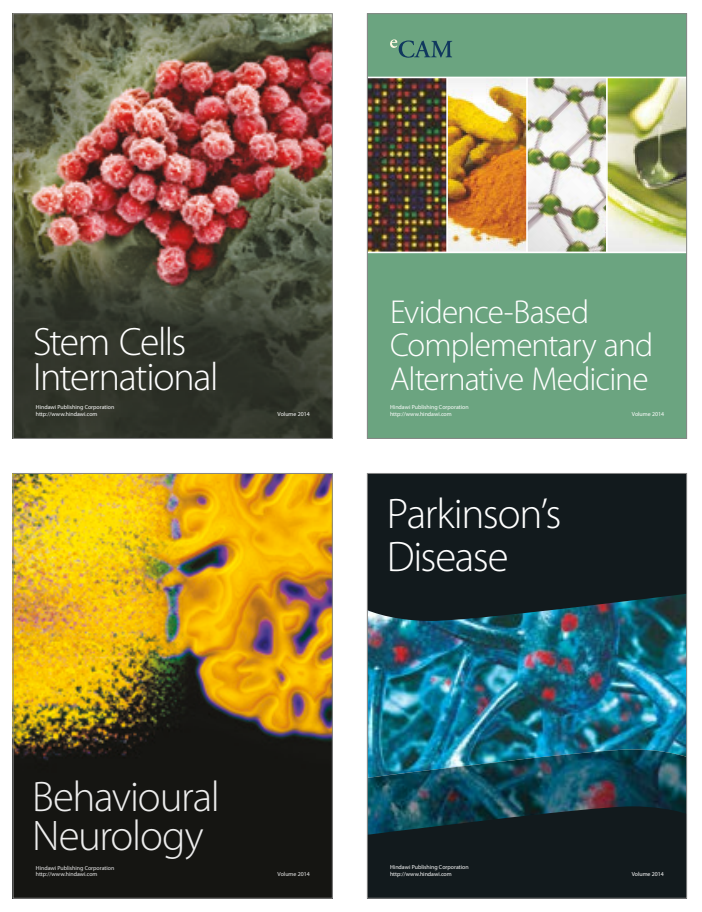
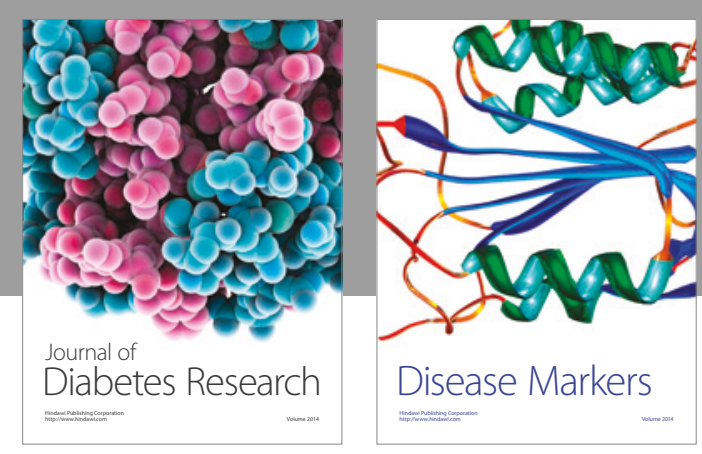

Disease Markers
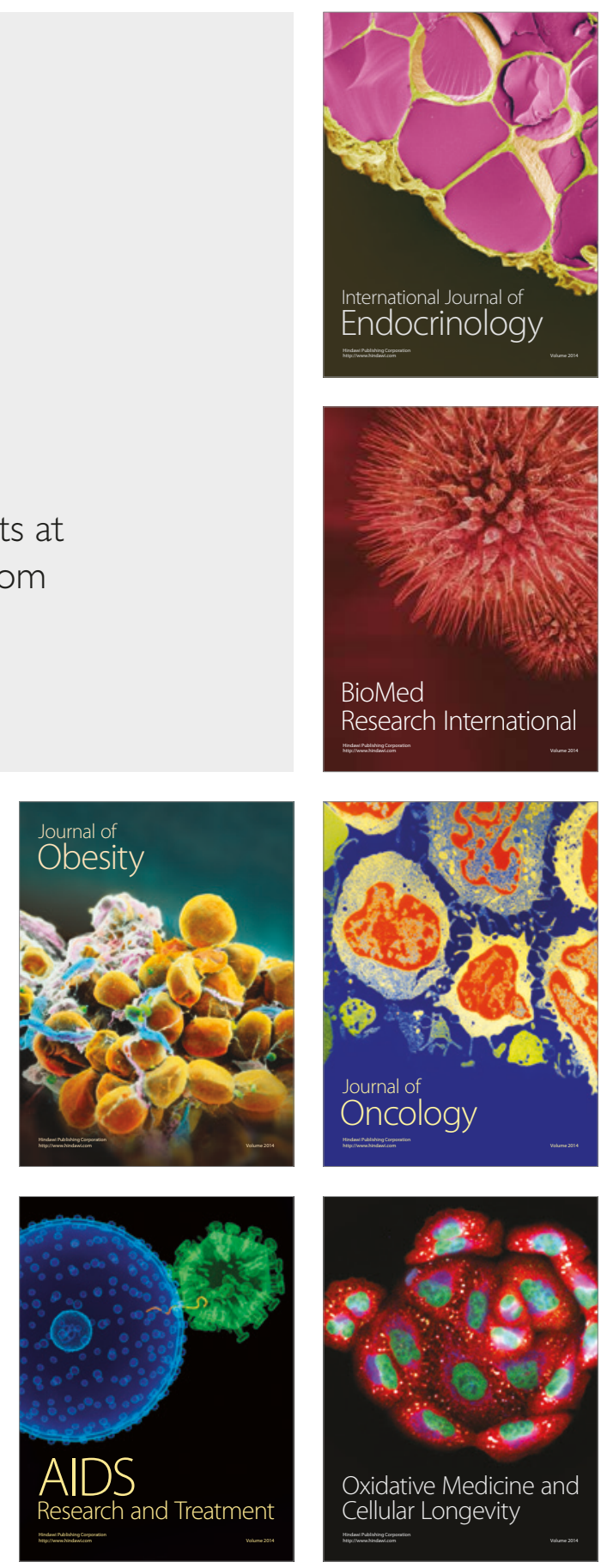